Check for updates

Cite this: RSC Adv., 2017, 7, 41098

Received 13th June 2017 Accepted 31st July 2017

DOI: $10.1039 / \mathrm{c} 7 \mathrm{ra06614g}$

rsc.li/rsc-advances

\section{PTMAc-PEG-PTMAc hydrogel modified by RGDC and hyaluronic acid promotes neural stem cells' survival and differentiation in vitro}

\author{
Ruirui Yang, $\dagger^{\mathrm{a}}$ Caixia $\mathrm{Xu}, \dagger^{\mathrm{a}}$ Tao Wang, ${ }^{\mathrm{b}}$ Yuanqi Wang, ${ }^{\mathrm{a}}$ Jingnan Wang, ${ }^{\mathrm{a}}$ \\ Daping Quant*b and David Y. B. Deng (ID $t^{* a c}$
}

\begin{abstract}
The enhancement of the biological properties of hydrogels by surface modifying with bioactive molecules is of great significance, especially for the treatment of central nervous system injury by combining engrafted cells. This study aimed to develop a novel polyethylene glycol (PEG)-based hydrogel modified by Arg-GlyAsp-Cys (RGDC) and hyaluronic acid (HA) as a carrier of neural stem cells (NSCs) for applications in central nervous system disorders. These novel gels were synthesized successfully and confirmed by GPC curve and ${ }^{1} \mathrm{H}$ NMR. The $\mathrm{G}^{\prime}$ increased with the increase in the contents of $H A$ in the blends and the pore size of the hydrogels was detected by SEM. We evaluated the adhesion, survival, and differentiation of NSCs on hydrogels modified by different proportions of RGDC and HA in vitro. The number of NSCs adherent on a $30 \%$ RGDC hydrogel was significantly higher than the number of NSCs adherent on $10 \%$ and $20 \%$ RGDC hydrogels. However, only $74 \%$ of the NSCs were viable in the $30 \%$ RGDC hydrogel. To further improve the survival rate of the NSCs, different proportions of HA were added to the $30 \%$ RGDC hydrogel to create Gel-1 (HA 33\%) and Gel-2 (HA 50\%). We found that both gel formulations, particularly Gel-1, increased the survival and differentiation rates of NSCs compared to the $30 \%$ RGDC hydrogel. Furthermore, Gel-1 exhibited low toxicity to NSCs, zebrafish embryogenesis, as well as the liver and kidneys in rats with spinal cord injuries at 1,4 , and 8 weeks. We conclude that this novel hydrogel (Gel-1) has good biocompatibility and can promote NSCs survival and differentiation into neurons. It could be used as a promising biological scaffold to treat central nervous system disorders in the future.
\end{abstract}

\section{Introduction}

Central nervous system diseases are considered a serious health problem, and many individuals suffer from disabilities such as Alzheimer's disease, Parkinson's disease, ${ }^{\mathbf{1}}$ stroke $^{2}$ and spinal

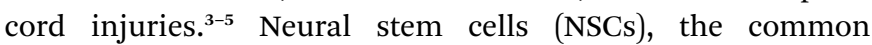
precursor cells of neurons and neural gliacytes, are the basis of the development of the nervous system and represent ideal cells for use in stem cell therapy. ${ }^{6-8}$ Some studies have investigated NSCs transplantation as a therapeutic strategy to mitigate the effects of a secondary injury in central nervous system disorders. ${ }^{\mathbf{9}, 10}$ However, when cells are simply directly delivered into the injury site, they are plagued by low rates of cell survival and

\footnotetext{
${ }^{a}$ Research Center of Translational Medicine, The First Affiliated Hospital, Sun Yat-sen University, Guangzhou 510080, China. E-mail: dengyub@mail.sysu.edu.cn

${ }^{b}$ PCFM Lab, GD HPPC Lab, Research Center of Engineering and Technology for Functional Biomaterials of Guangdong, School of Chemistry, Sun Yat-Sen University, Guangzhou 510275, China

'Guangdong Provincial Key Laboratory of Orthopedics and Traumatology, The First Affiliated Hospital, Sun Yat-sen University, Guangzhou 510080, China

$\dagger$ Both authors contributed equally to this work.

\$ Both corresponding authors contributed equally to this work.
}

poor engraftment because of hypoxic and ischemic environments. ${ }^{2,11,12}$ Successful cell-based therapy of central nervous system injuries highly depends on the survival of transplanted stem cells. Unprotected engrafted cells, in general, are associated with poor survival rates and low rates of differentiation into neurons. Therefore, alternatives are needed in order to efficiently deliver cells and mitigate the damage caused by the microenvironment for central nervous system disorders treatment. ${ }^{6,13,14}$

Approaches involving biomaterials as carriers for transplantation of stem cells, as well as tissue engineering approaches, have been in the forefront of new strategies for central nervous system injury treatment. ${ }^{\mathbf{1 3 , 1 5 - 1 7}}$ Among the biomaterials available, polyethylene glycol (PEG) basedhydrogels appear to be an excellent option, mainly due to their biocompatibility, non-immunogenicity, and physical properties which allow for precise control of the materials' mechanical properties. However, PEG is a relatively inert polymer and cells do not adhere to it nor survive well; it must therefore be modified in order to be used effectively. ${ }^{18,19}$ The bioactivity of PEG hydrogels can be improved by covalently introducing bioactive molecules such as peptides and proteins ${ }^{20}$ derived from native extracellular matrix (ECM). The short 
peptides Arg-Gly-Asp (RGD) motifs have been previously immobilized onto various substrates to promote the adhesion, differentiation, or neurite sprouting/outgrowth of NSCs. ${ }^{21,22}$ Hyaluronic acid (HA), one of the major components of native ECMs, has been used in remodeling and healing tissues and enables high cell proliferation and migration. ${ }^{17,23,24}$ In the central nervous system, HA is particularly abundant near neural stem cell niches and neuroblast migration routes (within the subventricular zone and rostral migratory stream, respectively). ${ }^{25}$ It has been reported that HA-based hydrogels support the survival, proliferation, and differentiation of neural stem

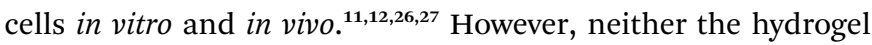
modified by ECM-derived peptides nor HA could be able to completely capture native ECM function and appropriately favor the survival of NSCs. ${ }^{28-30}$ Thus, we assume that the hydrogels modified by both peptides and HA could provide better support for the adhesion, survival, and differentiation of NSCs and better promote nerve tissue healing than those modified by a single factor.

In the present study, we first constructed a poly trimethylene carbonate/acryloyl cyclic carbonate-PEG-poly trimethylene carbonate/acryloyl cyclic carbonate (PTMAc-PEG-PTMAc, PTAE) copolymer. The side chain contains a large number of active double bonds, providing ample sites for the biological modification of the material. Afterwards, the PTAE copolymer was modified by Arg-Gly-Asp-Cys (RGDC), a covalently bound RGD analog, and HA, to construct a bio-functionalized injectable hydrogel encapsulating NSCs for the treatment of central nerve system injury. We tested the effect of the different proportions of RGDC and HA-modified hydrogel on the NSCs' adhesion, survival, and differentiation, and the toxicity of the extraction liquid in vitro and in vivo.

\section{Materials and methods}

\subsection{Materials}

PEG ( $M_{\mathrm{W}}$ : $10000 \mathrm{Da}$, Aldrich, Milwaukee, USA) was vacuum dehydrated for four hours at $120{ }^{\circ} \mathrm{C}$ prior to use. Trimethylene carbonate (TMC) (Huayang Medical Device Company, Huizhou, China) was recrystallized in ethyl acetate prior to use. Acryloyl cyclic carbonate (Ac) was synthesized as previously described. ${ }^{31}$ 1,8-Diazabicyclo undec-7-ene (DBU) and dithiothreitol (DTT) were purchased from J \& K Scientific (Shanghai, China). The other solvents were purchased from Guangzhou Chemical Reagent Factory (Guangzhou, China).

\subsection{The synthesis and characteristics of PTMAc-PEG-PTMAc copolymer and the modification by peptide addition}

The PTMAc-PEG-PTMAc polymer was synthesized in a two-step reaction via the ring-opening polymerization (ROP) of TMC and Ac using PEG as an initiator and DBU as the catalyst based on our previous report. ${ }^{32}$ The scheme of the reaction is shown in Scheme 1. A gel permeation chromatography (GPC) system (Waters 2959) was used to determine the molecular weight $\left(M_{\mathrm{n}}\right.$ $18.7 \mathrm{kD}$ and $M_{\mathrm{w}} 23.4 \mathrm{kD}$ ) and polydispersity (1.25 as calculated with the $M_{\mathrm{w}} / M_{\mathrm{n}}$ ) of the copolymers. Tetrahydrofuran (THF) was

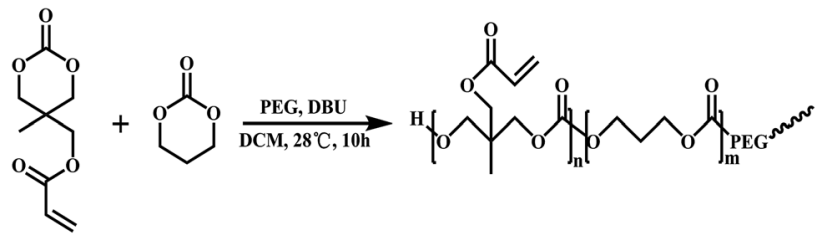

Scheme 1 Synthetic route of PTMAC-PEG-PTMAc.

used as the eluent at a flow rate of $1.0 \mathrm{ml} \mathrm{min}^{-1}$ at $40{ }^{\circ} \mathrm{C}$ and the $M_{\mathrm{w}}$ was calibrated using polystyrene standards.

PTMAc-PEG-PTMAc copolymer (abbreviated as PTAE, 100 $\mathrm{mg}$ ) was dissolved in $1.9 \mathrm{ml} \mathrm{NaH} \mathrm{PO}_{4} / \mathrm{Na}_{2} \mathrm{HPO}_{4}$ buffer (PBS, $\mathrm{pH}$ $7.4,0.1 \mathrm{M})$ at $37{ }^{\circ} \mathrm{C}$ overnight, which yielded $5 \mathrm{wt} \%$ polymer. Subsequently, a quantitative combination of RGDC peptide (arginine-glycine-aspartate-cysteine, 98\%) (GL Biochem, Shanghai, China) ([RGDC]/[Ac] $=3 / 10)$ was added and allowed to react for two hours under a nitrogen atmosphere. The peptide functionalized copolymer (abbreviated as PTAE + peptide) was obtained via lyophilisation after dialysis (MWCO 5000) in PBS at room temperature and was then dried under vacuum at $40{ }^{\circ} \mathrm{C}$ to a constant weight. The ${ }^{1} \mathrm{H}$ NMR spectra were used to confirm the modification of the peptide onto the PTAE. (Mercury-plus VARIAN, $300 \mathrm{MHz}$, DMSO-d6 as the solvent)

\subsection{The fabrication and characterization of hydrogels modified by peptide and $\mathrm{HA}$ addition}

$5 \%$ PTAE $+30 \%$ peptide aqueous solution and $2.5 \%, 5 \%(\mathrm{w} / \mathrm{w})$ HA were mixed respectively to form Gel-1 and Gel-2 (PTAE $+30 \%$ peptide: HA $=2: 1$ and $1: 1)$. Then, quantitative DTT ([DTT]/ $[\mathrm{Ac}]=3.5 / 10$ ) was added into the solution to react for 4 hours at $37{ }^{\circ} \mathrm{C}$ and the hydrogel was obtained. In the time sweep mode, the viscoelasticity of the hydrogels was analyzed with a rotating rheometer (ARES/RFS, TA, USA) with a parallel plate fixture (diameter, $25 \mathrm{~mm}$; gap, $1 \mathrm{~mm}$ ) at $37{ }^{\circ} \mathrm{C}$, which was adjusted by a bath temperature controller (Neslab). The polymer solution was quickly placed on the parallel plate immediately after the DTT was mixed. A plastic cover was used to minimize solvent evaporation. The storage modulus $\left(\mathrm{G}^{\prime}\right)$ and loss modulus $\left(\mathrm{G}^{\prime \prime}\right)$ of the hydrogels were recorded at a constant frequency $(10 \mathrm{~Hz})$ and strain $(1 \%)$ that was confirmed at the linear viscoelastic region. The internal structure of the hydrogels was examined with a scanning electron microscope (SEM, JEOL-7001F, Tokyo, Japan).

\subsection{NSCs Isolation and Culture}

Primary NSCs were obtained from fetal brains of E14 SpragueDawley (SD) rats based on previously published protocols and in accordance with a protocol approved by the Institutional Animal Care and Use Committee at Sun Yat-sen University. Briefly, the fetal brains were mechanically dissected and dissociated. Then, the cell suspension was centrifuged and the pelleted cells were resuspended in growth medium, which contained Dulbecco's Modified Eagle Media supplemented Nutrient Mixture F-12 (1:1) basic (Life Technologies, Carlsbad, 
CA), 2\% B27 (Life Technologies, Carlsbad, CA), 1\% L-glutamine (Gibco-Invitrogen), 1\% penicillin/streptomycin (Sigma), $20 \mathrm{ng} \mathrm{ml}^{-1}$ epidermal growth factor (EGF) (Peprotech, Carlsbad, CA), and $20 \mathrm{ng} \mathrm{ml}{ }^{-1}$ fibroblast growth factor (FGF)-2 (Life Technologies, Carlsbad, CA), and then cultured in a humidified incubator at $37{ }^{\circ} \mathrm{C}$ with $5 \% \mathrm{CO}_{2}$. The neurospheres were subcultured when the sizes reached $100 \mu \mathrm{m}$ in diameter. All NSCs used in this study were between passage 2 and 4 .

\subsection{NSCs seeding}

For two-dimensional (2D) culture, $60 \mu \mathrm{l}$ NSCs suspended at the concentration of $3 \times 10^{6}$ per $\mathrm{ml}$ were inoculated into the medium or on the surface of hydrogels containing different proportions of RGDC and HA. For three-dimensional (3D) culture, $200 \mu \mathrm{l}$ NSCs suspension with the same concentration was encapsulated in $1 \mathrm{ml}$ medium or different hydrogels. The resulting gel/cells constructs were cultured in appropriate media for 14 days. Growth medium was used for studies of cell attachment, viability and proliferation. Neuronal medium consisting of growth medium and 2\% FBS was used for differentiation studies. The medium was changed every 3 days. NSCs suspended at the same density were cultured in the wells of tissue culture plates as positive control.

\subsection{Cell adhesion}

Non-labeled NSCs were inoculated onto the surface of hydrogels for four hours. After rinsing the hydrogels with sterile PBS, adhesive NSCs were stained with calcein-AM (Life Technologies, Carlsbad, CA) and observed by a fluorescence microscope (IX73, Olympus, Japan). Cell counts were used to calculate cell density (cells per $\mathrm{cm}^{2}$ ) as a measure of cell attachment to the hydrogels.

\subsection{Cell viability and proliferation}

Cell viability was characterized by the Live/Dead assay. After three days in $2 \mathrm{D}$ culture, the cells were stained in $500 \mu \mathrm{l}$ culture media containing $2 \mathrm{mM}$ of calcein-AM for live cells and $4 \mathrm{mM}$ ethidium homodimer (EthD-I) for dead cells for 50 minutes at $37{ }^{\circ} \mathrm{C}$. Cell viability was calculated by determining the percentage of calcein-AM positive cells over the total number of cells in at least five randomly selected fields in each section. Positively stained cells were analyzed by using the particle counter plugin for Image $\mathrm{J}(\mathrm{NIH}$, Bethesda, $\mathrm{MD}$ ) and reported as percent viability.

For the study of NSCs proliferation in 3D gels, cells were labeled with the fluorescent carbocyanine membrane Dil dye (CM-DiL, molecular Probes, Eugene, Oregon, USA). Dil-labeled NSCs were cultured in 3D gels for 7 days. Cell proliferation was observed and analyzed by the fluorescence microscope (IX73, Olympus, Japan) using the particle counter plugin for Image $\mathrm{J}$ (NIH, Bethesda, MD) at 1, 3, 7 days.

\subsection{Cell differentiation}

Immunofluorescence analysis was performed to visualize the differentiation of cells. Non-labeled NSCs were cultured in 2D or
$3 \mathrm{D}$ gels with neuronal differentiation medium. At day 14 , the $2 \mathrm{D}$ construction was fixed for 20 minutes with $4 \%$ formaldehyde, followed by blocking with $2 \%$ BSA (Sigma) for 30 minutes. The cells were incubated with $\beta 3$-tublin $(1: 100)$ and glial fibrillary acidic protein (GFAP) (1:300) (CST, Boston, USA) antibody overnight at $4{ }^{\circ} \mathrm{C}$. Appropriate secondary antibodies were applied for one hour, then with $4^{\prime}$,6-diamidino-2-phenylindole dilactate (DAPI, Life Technologies, Carlsbad, CA) for five minutes to stain the nuclei. Fluorescence images were captured using a fluorescence microscope (IX-73, Olympus, Japan) and analyzed by Image J software.

Protein lysates were extracted from cells encapsuled in gels at day 14. Bicinchoninic acid (BCA) was used for protein quantification. $12 \%$ resolving and $8 \%$ stacking polyacrylamide gels (Solarbio Bio Inc., Shanghai, CHINA) with a Bio-Rad system (Bio-Rad, Shanghai, CHINA) were used to isolate target protein from $20 \mu \mathrm{g}$ of protein samples. Subsequently, proteins were transferred to PVDF membranes at $80 \mathrm{~V}$ for $60 \mathrm{~min}$ in ice cold transferring buffer and blocked in $5 \%$ skimmed milk at $4{ }^{\circ} \mathrm{C}$ overnight. The PVDF membranes were incubated with different antibodies at $4{ }^{\circ} \mathrm{C}$ overnight: GFAP (CST, USA, $\left.1: 1200\right) \beta 3$ tubulin (CST, USA, $1: 1000$ ) and incubated with Goat AntiRabbit IgG, AP Conjugated (Cwbio, CW0111S, CHINA, $1: 2200$ ) for $1.5 \mathrm{~h}$ at room temperature, washed in TBST and developed using an enhanced chemiluminescence (ECL) system (Millipore, USA). The relative protein expression was analyzed using VisionWorks LS of BioSpectrum Imaging System.

\subsection{Hydrogel toxicity test in vitro}

Gel-1 was immersed in artificial cerebrospinal fluid ${ }^{33}(119 \mathrm{mM}$ $\mathrm{NaCl}, 2.5 \mathrm{mM} \mathrm{KCl}, 1.3 \mathrm{mM} \mathrm{MgSO}$, $26.2 \mathrm{mM} \mathrm{NaHCO}_{3}, 1 \mathrm{mM}$ $\mathrm{NaH}_{2} \mathrm{PO}_{4}, 2.5 \mathrm{mM} \mathrm{CaCl}$, and $11 \mathrm{mM}$ glucose), with the $\mathrm{pH}$ maintained at 7.4, gassed with $95 \% \mathrm{O}_{2}-5 \% \mathrm{CO}_{2}$, and placed in a constant temperature incubator at $37^{\circ} \mathrm{C}$ for 100 days. The supernatant of Gel-1 degradation (extraction liquid) was obtained at day $1,7,14,28$, and 100 .

NSCs were cultured in a growth medium which contained $20 \%$ extraction liquid of Gel-1 for three days. NSCs cultured in medium containing $20 \%$ PBS were used as the control group. The sphere-forming ability of the NSCs was observed at day 3 . Then, $20 \%$ extraction liquid was added into the nutritive water of the zebrafish spawn and the zebrafish embryogenesis was observed continuously by taking photos after 96 hours. Nutritive water of the zebrafish spawn containing 20\% PBS (control group).

\subsection{Hydrogel toxicity test in vivo}

All experimental protocols and animal handling procedures were approved by the Animal Care and Use Committee of Sun Yat-sen University and were consistent with the National Institutes of Health Guide for the Care and Use of Laboratory Animals. No mortality occurred during these procedures.

Adult female Sprague \& Dawley (SD) rats (220-250 g, $n=20$ ) were randomly divided into 4 groups: control group $(n=5) ; 1 \mathrm{w}(n$ $=5) ; 4 \mathrm{w}(n=5) ; 8 \mathrm{w}(n=5)$. Briefly, animals were anesthetized with $1 \%$ pentobarbital sodium ( $40 \mathrm{mg} \mathrm{kg}^{-1}$, i.p.). A laminectomy was performed at the T9 vertebral level to expose the T9 and T10 
spinal segments. A $2 \mathrm{~mm}$ segment of spinal cord at the T10 level was completely removed. After hemostasis, Gel- 1 was injected respectively to fill up the gap. Postoperative animals were injected $40000 \mathrm{U}$ penicillin for 3 days to protect against infection as well as dehydration. Bladders were manually expressed twice daily until the urinary function was restored.

At $0,1,4$, and 8 weeks after transplantation, the rats were sacrificed. Liver and kidney tissues were fixed in 10\% neutral buffered formalin and embedded in paraffin. Tissue blocks were sectioned at $4 \mu \mathrm{m}$ in thickness, stained with hematoxylin and eosin (H\&E), and observed by light microscope (Olympus, Japan).

\subsection{Statistical analysis}

The results are expressed as mean \pm standard deviation. SPSS 19.0 statistical software was used for one way analysis of variance (ANOVA) (G') test, (cell adhesion, survival and differentiation) and repeated measurements of variance analysis (cell proliferation). Statistical significance was set to $p$-value $p<0.05$.

\section{Results and discussion}

\subsection{The synthesis of PTMAc-PEG-PTMAc copolymer and modification with peptide}

The PTMAc-PEG-PTMAc copolymers were synthesised by using the one-step ring-opening polymerisation (ROP) of TMC and Ac with PEG ${ }^{1} \mathrm{H}$ NMR and GPC were used to characterize the copolymers. The GPC curve (Fig. 1A) of PTAE showed an earlier elution time in contrast with PEG. In the ${ }^{1} \mathrm{H}$ NMR spectrum (Fig. 1B), characteristic peaks at 5.8-6.5 ppm, which were assigned to the double bonds, appeared. The results indicated that the copolymer with active pendent double bonds was successful synthesised.

The peptide modification onto the above copolymer via double bonds of the Ac segment was confirmed by ${ }^{1} \mathrm{H}$ NMR. It was shown that the typical resonance peaks of the protons of the double bonded carbons (5.8 to $6.5 \mathrm{ppm}$ ) disappeared almost completely after the Michael-type addition reaction with equivalent amounts of Ac and peptide, suggesting that the biomodification reaction using the RGDC peptide can be quantitatively controlled (Fig. 1B). The new resonances signals in the range of 8.0 to $9.0 \mathrm{ppm}, 2.6-2.7 \mathrm{ppm}$, and $1.4-1.6 \mathrm{ppm}$ appeared, and were attributed to RGDC peptide sequences.
A

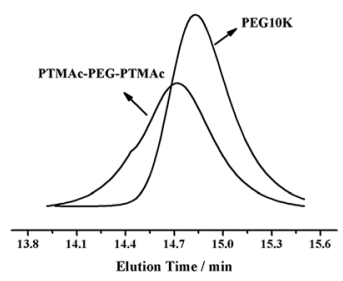

B

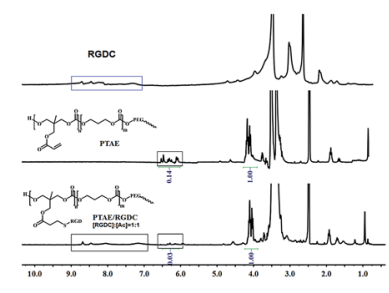

Fig. 1 (A) GPC curve of PEG and PTMAC-PEG-PTMAc copolymer; (B) ${ }^{1} \mathrm{H}$ NMR assessment for the PTMAC-PEG-PTMAc copolymer before and after modified by peptide.
A

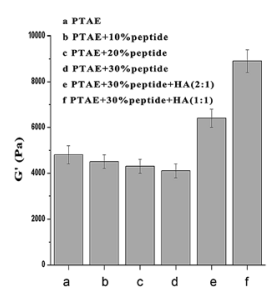

B

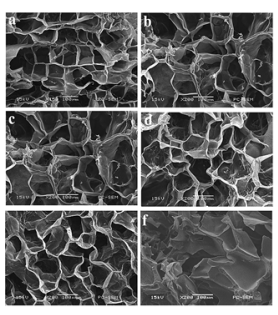

Fig. 2 (A) The correlation of the storage modulus with the composition of the hydrogels based on the PTMAc-PEG-PTMAc copolymer; (B) SEM observation of the hydrogel with different components (scale bar $=100 \mu \mathrm{m}$ ).

\subsection{The properties of hydrogels}

As shown in Fig. 2A, the $\mathrm{G}^{\prime}$ of the hydrogels based on PTAE changed a little before and after peptide modification with different ratios $(10 \%, 20 \%, 30 \%)$, which remained in the range of 4 to $5 \mathrm{KPa}$. However, the $\mathrm{G}^{\prime}$ increased with the increase in the contents of $\mathrm{HA}$ in the blends, i.e., $6.3 \mathrm{KPa}$ for Gel-1 (PTAE/HA = 2/1) and 9.0 KPa for Gel-2 (PTAE/HA = 1/1). Kang MiLan et al. reported that the higher HA concentrations resulted in a more highly cross-linked structure, which displayed greater viscosity and viscoelasticity. ${ }^{34}$ It was also found that there were no obvious changes in the pores size of the hydrogels before or after peptide modification, and the average pore sizes ranged from several micrometers to several hundreds of micrometers (Fig. 2B). Incorporation of lower HA (Gel-1) resulted in more regular porous structures, although the pores sizes of Gel-1 had no obvious change compared to those of the hydrogels modified by peptide addition. Differently, the incorporation of higher HA (Gel-2) obviously increased the pores sizes of hydrogel and resulted in irregular porous structures (Fig. 2B-F). According to previous studies, ${ }^{22}$ the pore size was directly influenced by the cross-linking density, which related to the density of amino groups and aldehyde groups of the hydrogel.
A

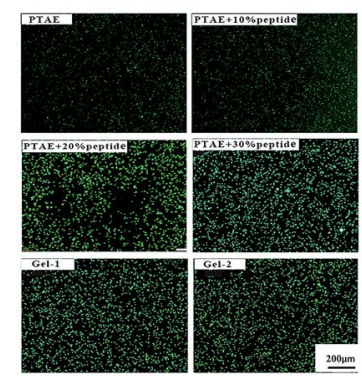

B

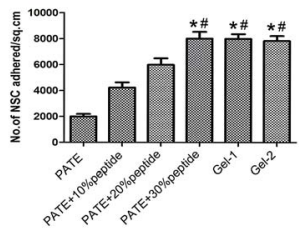

Fig. 3 Cell adhesion on hydrogels modified by different portions of peptide and HA after 4 hour incubation. (A). Representative images showing adhesive NSCs stained with calcein AM in different modified or unmodified PTAE hydrogels. (B) Number of cells adhered per unit area, as determined by counting calcein AM-stained cells. Adhesion of $30 \%$ peptide-PTAE and Gel-1 surfaces was significantly greater than the PTAE control, $10 \%$ and $20 \%$ peptide-PTAE, and Gel-2. All values are expressed as mean \pm SEM $\left(n=5,{ }^{*} p<0.05,{ }^{*} p<0.01\right)$ (scale bars $=100 \mu \mathrm{m}$ ). 


\subsection{Influence of RGDC on cell adhesion in vitro}

The results of cell adhesion are shown in Fig. 3. Compared with the PTAE group, more cells adhered to the gels modified with the RGDC peptide in a concentration dependent manner (Fig. 3A). The 30\% RGDC hydrogel ( $8120 \pm 124$ NSCs per sq.cm) showed significantly greater cell adhesion compared to the $10 \%$ and $20 \%$ RGDC hydrogels (4001 \pm 173 NSCs per sq cm, $6000 \pm$ 140 NSCs per sq $\mathrm{cm}, p<0.05)$. Furthermore, there was no significant difference between cells cultured in Gel-1 and the $30 \%$ RGD hydrogel. At the same time, the number of cells grown in Gel-2 was lower than that in grown in the $30 \%$ RGDC hydrogel (Fig. 3B). Cell adhesion, directly influencing cell proliferation and differentiation on scaffolds, is a crucial indicator for scaffold bioactivity. ${ }^{\mathbf{1 5 , 3 4}}$ The RGDC binding domain is incorporating peptide binding motifs, which within hydrogels to promote cell adhesion. ${ }^{35-37}$ In this study, with the increasing of concentration of RGDC $(10 \%, 20 \%$, and $30 \%)$, more NSCs adhered onto the gel compared to the PTAE group. This result is in accordance with previous study results. It has been reported that RGDC enhanced cell adhesion via integrin-mediated signaling. ${ }^{38}$ However, the inclusion of $33 \%$ HA did not increase NSCs adhesion; instead, 50\% HA decreased NSCs adhesion compared to $30 \%$ peptide gel. The possible reason is that HA is an extremely long, negatively charged glycosaminoglycan that could cause the normal cell membrane charge repulsion, leading to less cell adhesion. ${ }^{29,39}$

\subsection{Influence of HA on cell survival and proliferation in vitro}

The survival rate of NSCs in 30\% RGDC hydrogel, Gel-1, and Gel2 was $77 \pm 0.21 \%, 93 \pm 0.23 \%$ and $83 \pm 0.14 \%$, respectively (Fig. 4A and C). The percentage of live cells in the Gel-1 group is significantly higher than that of the other two groups $(p<0.05)$. These results suggest that Gel-1 created the optimal microenvironment for the survival of NSCs.
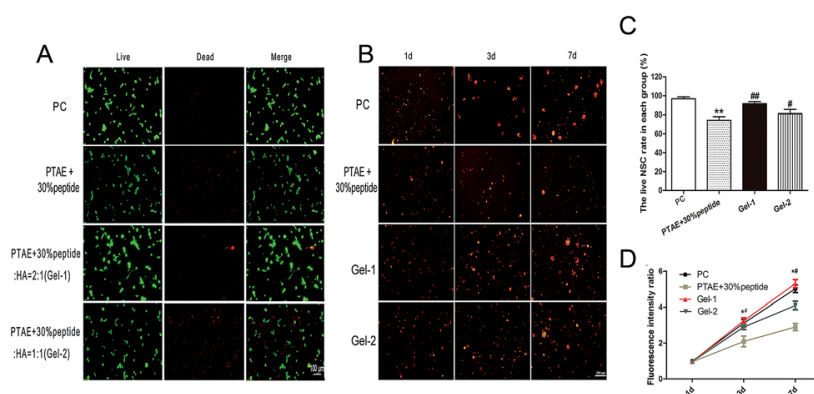

Fig. 4 Cell survival on hydrogels at day 3 in vitro and cell proliferation in 3D culture for 7 days. (A) Live/dead images showing live cells stained with calcein-AM (green) and dead cells stained with EthD-III (red) (B) fluorescent image of Dil labled NSCs (red) in medium, PTAE + 30\% peptide, Gel-1 and Gel-2 for 7 days. (C) Cell viability was calculated by determining the percentage of calcein-AM positive cells over the total number of cells. The percentage of live cells in the Gel-1 group is significantly higher than that of the other groups. (**p $<0.01$ vs. PC, ${ }^{\# \#} p<0.01$ vs. PTAE $+30 \%$ peptide, ${ }^{\#} p<0.05$ vs. PTAE $+30 \%$ peptide, scale bars $=100 \mu \mathrm{m}$ ). (D) Quantitative analysis of the NSCs proliferation by fluorescence intensity $\left(n=6,{ }^{*} p<0.05\right.$ vs. PC, ${ }^{\#} p<0.05$ vs. PTAE $+30 \%$ peptide scale bars $=200 \mu \mathrm{m}$ ). PC: postive control, cells in medium
At day 1, the NSCs in 3D culture were spherical and scattered in the hydrogels. At day 3 and 7 after seeding, cell spheres in all groups accreted gradually (Fig. 4B). The results of the cell quantification showed that proliferation among all four groups shared similar trends. As time went on, the number of NSCs on each gels increased gradually. Cell proliferation was significantly higher in the Gel-1 as compared to the 30\% RGDC hydrogel and Gel-2 at day 3 and $7(p<0.01)$. There was no significant difference between Gel-1 and the positive control (Fig. 4C and D). Interestingly, while the inclusion of HA did not increase the NSCs' adhesion, it significantly increased the survival rate of the NSCs compared to the $30 \%$ peptide gel (Fig. 4), especially the gel containing 33\% HA (Gel-1). In addition, Gel-1 obviously promoted NSCs' proliferation compared to the $30 \%$ peptide gel or Gel-2 in $3 \mathrm{D}$ culture. There are a couple of reasons for this. Firstly, HA has been confirmed to promoting cell proliferation and migration by interacting with cell surface receptors such as CD44. ${ }^{40}$ Secondly, given that the HA increased the storage modulus of the Gel (Fig. 2), which was also demonstrated by other similar study, ${ }^{28}$ it can be postulated that the mechanical properties of the low HA gels (Gel-1) are more conducive to the maintenance of NSCs culture than those of the higher HA content gels or simple $30 \%$ peptide gel. Thirdly, the network of Gel-1 kept the regular porous structures which enabled a more efficient transportation of nutrients and wastes throughout the hydrogel scaffolds, while the addition of HA in high concentration (Gel-2) resulted in the collapse of the regular porous network.

\subsection{NSCs differentiation within hydrogels}

At day 14 , the percentages of $\beta 3$-tublin-positive cells on $30 \%$ peptide hydrogel, Gel-1, and Gel-2 were determined to be

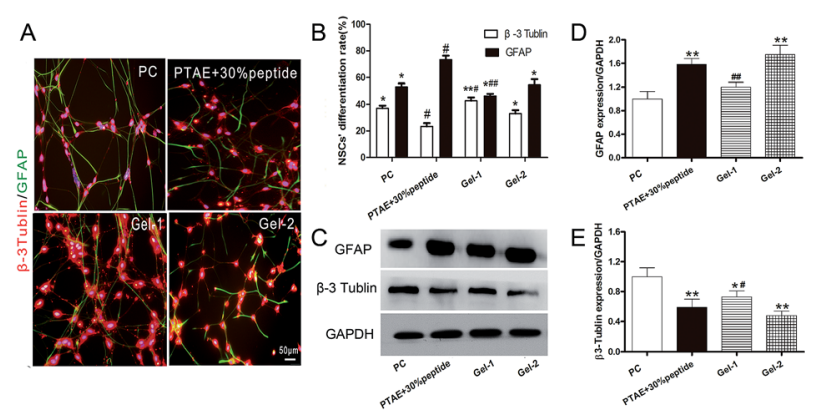

Fig. 5 Differentiation of NSCs cultured in 2D and 3D hydrogels with differential media for 14 days in vitro. (A) The expression of $\beta 3$-tublin and GFAP was detected by immunofluorescence staining in $2 D$ culture. $\beta 3$-Tublin for neurons appears as red, GFAP for astrocytes appears as green, and the nuclei that were stained with DAPI appear as blue. (B) The percentages of $\beta 3$-tublin-positive cells on Gel-1 were higher than those on 30\% peptide hydrogel or Gel-2, while GFAPpositive cells on Gel-1 were the lowest among all the groups. Scale bars $=50 \mu \mathrm{m}, * p<0.05$; $* p<0.01 n=5$. (C) The protein level of GFAP and $\beta 3$-tublin was detected by Western blot after NSCs were cultured in 3D Gels. (D) Quantification of protein levels of GFAP and (E) $\beta 3$ tublin normalized to GAPDH. Error bars represent the means \pm SD, PC: postive control, cells in medium. ( $n=3, p<0.05$ vs. PTAE $+30 \%$ peptide, ${ }^{\#} p<0.05$ vs. Gel-2, ${ }^{* *} p<0.01$ vs. PTAE $+30 \%$ peptide, ${ }^{\# \#} p<$ 0.01 vs. Gel-2). 
22.38\%, 43.74\%, 26.85\%, respectively (Fig. 5). Compared with the $30 \%$ peptide hydrogel and Gel-2, the percentages of GFAPpositive cells in Gel-1 were lower (Fig. 5B). GFAP is a marker of astrocyte and the decreased percentage of GFAP positive cells suggests that Gel-1 may promote or facilitate NSCs' neuronal differentiation.

Western blot analysis revealed after NSCs were encapsuled in $3 \mathrm{D}$, the expression of GFAP in Gel-1 was significantly lower than that in PTAE $+30 \%$ peptide and Gel-2. Nevertheless, the expression of $\beta 3$-tublin was significantly higher in Gel-1 at day 14 (Fig. 5C-E). Previous studies revealed that surface-bound RGD peptide could effectively enhance neuronal differentiation of NSCs and facilitate neurite outgrowth. ${ }^{21,41}$ In addition, it has been reported that HA plays an important role in neural commitment, $^{29}$ based on its role as a major extracellular component during fetal development. ${ }^{42}$ Therefore, as well as survival and proliferation, Gel-1 can also have more positive effects on NSCs differentiation than Gel-2 or $30 \%$ peptide gel (Fig. 5). Thus, we demonstrated that the PTAE hydrogel containing $30 \%$ peptide and $33 \%$ HA provided the most appropriate microenvironment for the adhesion, survival, and differentiation of NSCs in vitro.

\subsection{Gel-1 with low toxicity to cells and zebrafish embryogenesis}

Biomaterials must undergo strict qualified toxicology tests before clinical application. We first studied the toxicology of hydrogel extraction liquid to cells. As shown in Fig. 6A and B, quantification of the number of spheres formed from every 10000 cells demonstrated no neurosphere-forming defects in the six groups. Zebrafish, as an in vivo animal model, is considered as a more definitive assessment of toxicity, and it can clearly show the embryo toxicity. ${ }^{42}$ Similarly, the $20 \%$

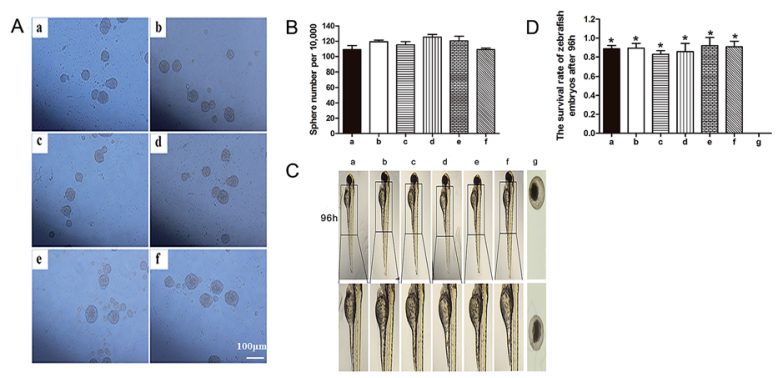

Fig. 6 Gel-1 with low toxicity to cells and zebrafish embryogenesis. (A) The different extraction liquid of Gel-1 was obtained at days 1 (b), 7 (c), 14 (d), 28 (e), and 100 (f). Sphere-forming of NSCs cultured in medium containing $20 \%$ extraction liquid from different days was detected at 72 h. NSCs cultured in medium containing $20 \%$ PBS represents the control group (a). The sphere-forming number from every 10000 cells demonstrates no neurosphere-forming defect in the six groups (a-f and B). (C) The effect of different extraction liquid concentration of Gel-1 (a-e) on zebrafish embryogenesis was assayed. Nutritive water of the zebrafish containing 20\% DMSO represents the control group (g). (D) The $20 \%$ extraction liquid of Gel-1 (a-e) had no detrimental effects on the zebrafish embryogenesis. Example (f) was exposed to DMSO which resulted in Zebrafish embryo dying and this material was proven toxic $* p<0.05$ vs. (f).

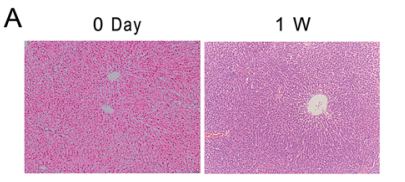

$4 \mathrm{~W}$

Liver

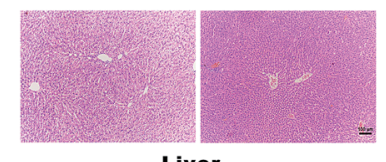

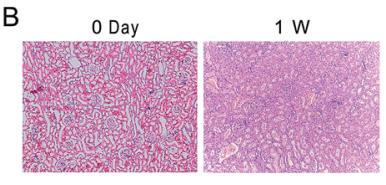

$4 \mathrm{~W}$

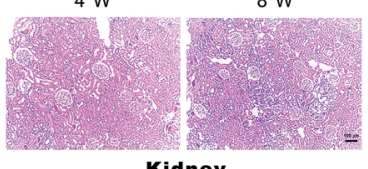

Kidney
Fig. 7 Gel-1 with low toxicity in vivo. Liver (A) and kidney (B) slices were stained using $\mathrm{HE}$. At week 1, 4, 8 post-surgery, the hepatic lobules and glomeruli of all rats retained complete structure $(n=5$, scale bars $=100 \mu \mathrm{m}$ ).

extraction liquid of Gel-1 had no detrimental effects on the zebrafish embryogenesis (Fig. 6C and D). These results preliminarily showed that Gel-1 had good biocompatibility.

\subsection{Gel-1 with low toxicity in vivo}

Because this novel synthetic biomaterial is potentially applied to the central nervous system injury, we had chosen a model spinal cord injury with higher incidence to evaluate the toxicity of Gel-1 in vivo. ${ }^{\mathbf{6} 11,13,15,17,43}$ At $0,1,4$, and 8 weeks post-surgery, we observed the hepatic lobules and glomeruli of spinal cord injured rats (Fig. 7). The liver and kidney structures had no obvious changes. Since the liver and kidneys are the most important organs for elimination of drugs and their metabolites, renal toxicity and hepatotoxicity are one of the most common adverse reactions during drug treatment. ${ }^{44}$ It would tremendously deteriorate a patient's condition if the acute toxic effects occurred in hepatic cells and the renal epithelial cells after the implantation of biomaterials. ${ }^{45}$ So, it is essential to research the toxicity of liver and kidney well. Unfortunately, because of the time limit, observation took place over only 8 weeks. Long term toxicity of implants was not assessed in SD rats in our present study by reason of the prohibitive costs. Long term safety profile studies of the gel need to be investigated for clinical applications.

\section{Conclusion}

We successfully fabricated a novel PTAE-based hydrogel modified by $30 \%$ RGDC and 33\% HA. The hydrogel has good biocompatibility and low toxicity. It could provide an ECM-like microenvironment supporting the survival and differentiation of NSCs in vitro. In addition, the modified hydrogel can be injected into injured spinal cords directly, so the application is more convenient. Therefore, Gel-1 could be used as a promising biological scaffold to treat central nervous system disorders in the future.

\section{Conflicts of interest}

There are no conflicts to declare. 


\section{Acknowledgements}

This work was supported by the National Key Research and Development Plan of China (No. 2016YFC1100103), the National Natural Science Foundation of China (Grant No. 81571105, No. 81472069, No. 81472039) and Natural Science Foundation Projects of Guangdong Province (Grant No. S2013020012818, 2015A030311025 and 2015A030313186). Thank you Luming Chen, Krsna Muscheck, Francis Tintani, for our English editing work on this manuscript.

\section{Notes and references}

1 D. H. Choi, J. H. Kim, S. M. Kim, K. Kang, D. W. Han and J. Lee, Int. J. Mol. Sci., 2017, 18, DOI: 10.3390/ijms18010224.

2 L. Wei, Z. Z. Wei, M. Q. Jiang, O. Mohamad and S. P. Yu, Prog. Neurobiol., 2017, DOI: 10.1016/j.pneurobio.2017.03.003.

3 D. Szczepanek, E. Wasik-Szczepanek, F. Stoma, B. Sokolowska and T. Trojanowski, Neurol. Neurochir. Pol., 2017, 51, 319-323.

4 A. M. Cochrane, C. Cheung, K. Rangan, D. Freyer, L. Nahata, G. Dhall and J. L. Finlay, Pediatr. Blood Cancer, 2017, DOI: 10.1002/pbc.26616.

5 D. A. Nader and Z. M. Sanchez, Am. J. Drug Alcohol Abuse, 2017, 1-15, DOI: 10.1080/00952990.2017.1306746.

6 S. Tang, X. Liao, B. Shi, Y. Qu, Z. Huang, Q. Lin, X. Guo and F. Pei, PLoS One, 2014, 9, e107517.

7 W. Sun, T. Incitti, C. Migliaresi, A. Quattrone, S. Casarosa and A. Motta, J. Tissue Eng. Regener. Med., 2017, 11, 15321541.

8 L. Truong, S. L. Harper and R. L. Tanguay, Methods Mol. Biol., 2011, 691, 271-279.

9 R. Gonzalez, M. H. Hamblin and J. P. Lee, CNS Neurol. Disord.: Drug Targets, 2016, 15, 881-886.

10 Q. Wang, F. Duan, M. X. Wang, X. D. Wang, P. Liu and L. Z. Ma, Clin. Neurol. Neurosurg., 2016, 146, 1-11.

11 A. J. Mothe, R. Y. Tam, T. Zahir, C. H. Tator and M. S. Shoichet, Biomaterials, 2013, 34, 3775-3783.

12 Y. Liang, P. Walczak and J. W. Bulte, Biomaterials, 2013, 34, 5521-5529.

13 R. C. Assuncao-Silva, E. D. Gomes, N. Sousa, N. A. Silva and A. J. Salgado, Stem Cells Int., 2015, 2015, 948040.

14 J. A. Burdick, M. Ward, E. Liang, M. J. Young and R. Langer, Biomaterials, 2006, 27, 452-459.

15 M. M. Pakulska, B. G. Ballios and M. S. Shoichet, Biomed. Mater., 2012, 7, 024101.

16 T. Russo, M. Tunesi, C. Giordano, A. Gloria and L. Ambrosio, Proc. Inst. Mech. Eng., Part H, 2015, 229, 905-916.

17 A. Agbay, J. M. Edgar, M. Robinson, T. Styan, K. Wilson, J. Schroll, J. Ko, N. Khadem Mohtaram, M. B. Jun and S. M. Willerth, Cells Tissues Organs, 2016, 202, 42-51.

18 S. L. Chen, R. H. Fu, S. F. Liao, S. P. Liu, S. Z. Lin and Y. C. Wang, Cell Transplant., 2017, DOI: 10.3727/ $096368917 \times 695380$.

19 A. S. Ahmed, U. K. Mandal, M. Taher, D. Susanti and J. M. Jaffri, Pharm. Dev. Technol., 2017, 1-10, DOI: 10.1080/ 10837450.2017.1295067.
20 Y. Luo and M. S. Shoichet, Nat. Mater., 2004, 3, 249-253.

21 H. Xie, J. Li, L. Li, Y. Dong, G. Q. Chen and K. C. Chen, Acta Biomater., 2013, 9, 7845-7854.

22 P. Naghdi, T. Tiraihi, F. Ganji, S. Darabi, T. Taheri and H. Kazemi, J. Tissue Eng. Regener. Med., 2016, 10, 199-208.

23 J. Schiavi, L. Reppel, N. Charif, N. de Isla, D. Mainard, N. Benkirane-Jessel, J. F. Stoltz, R. Rahouadj and C. Huselstein, J. Tissue Eng. Regener. Med., 2017, DOI: 10.1002/term.2461.

24 J. Gaston and S. L. Thibeault, Biomatter, 2013, 3, e23799.

25 M. H. El-Dakdouki, K. El-Boubbou, D. C. Zhu and X. Huang, RSC Adv., 2011, 1, 1449-1452.

26 K. T. Dicker, L. A. Gurski, S. Pradhan-Bhatt, R. L. Witt, M. C. Farach-Carson and X. Jia, Acta Biomater., 2014, 10, 1558-1570.

27 D. Shendi, D. R. Albrecht and A. Jain, J. Biomed. Mater. Res., Part A, 2017, 105, 608-618.

28 A. Ranga, M. P. Lutolf, J. Hilborn and D. A. Ossipov, Biomacromolecules, 2016, 17, 1553-1560.

29 K. Farrell, J. Joshi and C. R. Kothapalli, J. Biomed. Mater. Res., Part A, 2017, 105, 790-805.

30 B. G. Ballios, M. J. Cooke, L. Donaldson, B. L. Coles, C. M. Morshead, D. van der Kooy and M. S. Shoichet, Stem Cell Rep., 2015, 4, 1031-1045.

31 W. Chen, H. C. Yang, R. Wang, R. Cheng, F. H. Meng, W. X. Wei and Z. Y. Zhong, Macromolecules, 2013, 14, 1214-1222.

32 T. Wang, M. Yan, X. Sun and D. Quan, Polymer, 2015, 57, 21-28.

33 S. B. Wong, S. J. h. Cheng, W. C. Hung, W. T. Lee and M. Y. Min, PLoS One, 2015, 10, 1-14.

34 M. L. Kang, S. Y. Jeong and G. I. Im, Tissue Eng., Part A, 2017, 3, 524-532.

35 C. C. Lin, RSC Adv., 2015, 5, 39844-398583.

36 R. Gaetani, D. A. Feyen, V. Verhage, R. Slaats, E. Messina, K. L. Christman, A. Giacomello, P. A. Doevendans and J. P. Sluijter, Biomaterials, 2015, 61, 339-348.

37 Y. Liu, H. Ye, K. Satkunendrarajah, G. S. Yao, Y. Bayon and M. G. Fehlings, Acta Biomater., 2013, 9, 8075-8088.

38 M. Baiula, P. Galletti, G. Martelli, R. Soldat, L. Belvisi, M. Civera, S. D. Dattoli, S. M. Spampinato and D. Giacomini, J. Med. Chem., 2016, 59, 9721-9742.

39 K. Xu, K. Narayanan, F. Lee, K. H. Bae, S. Gao and M. Kurisawa, Acta Biomater., 2015, 24, 159-171.

40 X. Zhang, P. Zhou, Y. Zhao, M. Wang and S. Wei, Carbohydr. Polym., 2016, 136, 1061-1064.

41 A. C. de Luca, A. Faroni, S. Downes and G. Terenghi, J. Tissue Eng. Regener. Med., 2016, 10, 647-655.

42 S. K. Seidlits, Z. Z. Khaing, R. R. Petersen, J. D. Nickels, J. E. Vanscoy, J. B. Shear and C. E. Schmidt, Biomaterials, 2010, 31, 3930-3940.

43 N. A. Silva, N. Sousa, R. L. Reis and A. J. Salgado, Prog. Neurobiol., 2014, 114, 25-57.

44 L. Y. Rzzo, S. K. Golombek, M. E. Mertens, Y. Pan, D. Laaf, J. Broda, J. Jayapaul, D. Mockel, V. Subr, W. E. Hennink, G. Storm, U. Simon, W. Jahnen-Dechent, F. Kiessling and T. Lammers, J. Mater. Chem. B, 2013, 1, 3918-3925.

45 A. J. Hill, H. Teraoka, W. Heideman and R. E. Peterson, Toxicol. Sci., 2005, 86, 6-19. 